\title{
RNA-like polymer model: exact calculation on the Bethe lattice
}

\author{
M. Pretti \\ Consiglio Nazionale delle Ricerche - Istituto Nazionale per la Fisica della Materia (CNR-INFM) \\ Dipartimento di Fisica, Politecnico di Torino, Corso Duca degli Abruzzi 24, I-10129 Torino, Italy
}

(Dated: August 6, 2018)

\begin{abstract}
We consider a lattice polymer model (random walk), in which the walk is allowed to visit lattice bonds at most twice. Such a model might have some relevance to describe statistical properties of RNA molecules. In order to mimic base pairing, we assign an attractive energy term to each doublyvisited bond, and a further contribution to each pair of consecutive doubly-visited bonds. The latter term is expected to mimic the stacking effect, whereas no effect of sequence, that is, of chemical specificity, is taken into account. The phase diagram is worked out exactly on a Bethe lattice, in a grand-canonical formulation. In the single molecule limit, the system undergoes two different phase transitions, upon decreasing temperature: a $\Theta$-like collapse from a swollen "coil" state to a "molten" state, with a low fraction of doubly-visited bonds, and subsequently to a "paired" state, with empty or doubly-visited bonds only. The stacking effect drives the latter transition from second to first order.
\end{abstract}

PACS numbers: $\quad$ 05.70.Fh, 64.60.Cn, 61.25.Hq, 87.15.Aa, 87.14.Gg

\section{INTRODUCTION}

Lattice self-avoiding walks, i.e., random walks that are forbidden to visit lattice sites more than once, have long been employed for modeling linear polymers in a good solvent [1]. A short range interaction between nonconsecutive monomers has been also considered, in order to represent either Van der Waals attractive forces between monomers or the effective result of solvophobic interactions. Such interactions cause the well-known $\Theta$ transition from a swollen coil at high temperature to a compact globule at low temperature [2]. The Bethe approximation [3, 4, 5], i.e., the exact solution on the Bethe lattice, has been shown to reproduce with reasonable accuracy, and with negligible computational effort, the phase behavior of such basic model $(\Theta$ model $)$ and also of slightly more complicated polymer models [6, 7, 8, $, 9,10]$.

Several variations of the $\Theta$ model have been proposed, in order to describe different physical phenomena. In particular, it is possible to relax the self-avoidance constraint, allowing the walk to visit lattice bonds at most twice. If the polymer chain is assigned an orientation, and lattice bonds are allowed to be doubly-visited only by opposite chain segments, the model is denoted as 2tolerant trail 11, 12. This model may be useful to investigate configurational statistics of RNA molecules, whose importance in molecular biology is being more and more recognized [13, 14, 15, 16]. Similar to DNA, a RNA molecule is a long polymer chain composed of four different monomers (bases), adenine, cytosine, guanine, and uracil, which are pairwise complementary (i.e., adenine-uracil and cytosine-guanine pairings are energetically favored by formation of hydrogen bonds [17]). At a coarse-grained level, one can neglect the differences among bases, and assign an attractive (contact) energy for each base pairing, that is, for each doubly-visited bond.

Quite recently, Baiesi, Orlandini, and Stella [1] have investigated the previously described model, performing accurate Monte Carlo simulations on the face-centered cubic (fcc) lattice, fully taking into account the excluded volume effect, and showing the existence of a continuous phase transition (similar to the $\Theta$ collapse) from a high temperature state in which the RNA is almost completely unpaired to a low temperature state with a significant fraction of paired bases (the so-called molten phase).

In this work, we first verify that the Bethe approximation, which is able to take into account excluded volume at a local level 9], predicts a $\Theta$-like transition as well. Moreover, we consider an extended model with a more general energy function: We assign a specific energy contribution to consecutive paired bases, without intermediate branching, in order to mimic the so-called stacking effect 17, 18, 19]. Indeed, the stacking effect, mostly related to hydrophobicity [18], is claimed to be energetically more relevant than base pairing [17], and however has great importance for algorithms attempting to predict the secondary structure of given RNA sequences [20]. In the statistical physics literature, models with ordinary pairing energy only [11, 12, 21, 22, 23, 24], or with stacking energy only 25], or both [26 have been considered, but the relative importance of the stacking effect with respect to base pairing has been scarcely investigated 27. On the contrary, we specifically address the issue of stacking, assigning different relevance to one of the two interactions, by means of an adjustable parameter. We observe that our model predicts, in the low temperature region, a fully base-paired phase. Such phase, which does not at all correspond to a unique secondary structure, might describe -with some cautions- an average "native" state. The phase transition to the molten phase ("denaturation") turns out to be continuous, for the ordinary pairing energy model, but, upon adding even a small stacking energy, it turns out to change into first order.

The paper is organized as follows. In Sec. II] we intro- 
duce the model in some more detail and give an overview of the Bethe lattice calculation. In Sec. [III we work out the phase behavior of the model, with particular attention to the single-molecule limit, and in Sec. IV] we discuss the results, adding some concluding remarks. Appendices $\mathrm{A}$ and $\mathrm{B}$ are devoted respectively to a derivation of the equilibrium free energy and of the recursion equations for the Bethe lattice, while in Appendix [C] we report the analytical calculation of the $\Theta$-like transition temperature.

\section{THE MODEL AND THE BETHE LATTICE CALCULATION}

As previously mentioned, our polymer model is a selfavoiding walk, which is exceptionally allowed to visit each lattice bond (at most) twice, but is not allowed to self-intersect. We can imagine roughly the following picture. Each segment of the walk represents a monomer (base), whereas empty lattice bonds represent the solvent. Doubly-visited bonds (i.e., bonds occupied by two segments) represent paired bases, yielding an attractive energy $-(\beta-\gamma)$, with $\beta>\gamma>0$. Moreover, every pair of consecutive doubly-visited bonds yields an additional attractive contribution $-\gamma$, providing a rough description of the stacking effect. It turns out that $\beta$ is the maximum pairing energy, obtained by consecutive basepairing, and will be taken as the energy unit. According to the grand-canonical formulation, a chemical potential $\mu$ is associated to each monomer, while the solvent chemical potential is conventionally assumed to be zero.

Let us spend a few words to notify that the coarsegrained model introduced above includes some degree of inconsistency. In particular, to be more precise, segments of the walk ought to represent stretches of the order of the persistence length of the polymer chain. Unfortunately, the persistence length turns out to be much different for single- or double-stranded RNA stretches (being much larger in the latter case), but we nonetheless describe both cases within a single lattice bond. We expect that such inconsistency should not alter the qualitative phase behavior of the model, since this is what happens for similar models of DNA, as it has been also noted in Ref. 11 .

A configuration of the system can be defined by specifying the number of segments on each lattice bond. Therefore, we define a configuration variable $n_{i}=0,1,2$ (occupation number) for the $i$-th lattice bond. Of course, such configuration variables are not independent, but have to satisfy some constraints. In particular, on each set of bonds of a given site, we impose the following conditions: (i) the total number of segments must be even; (ii) there cannot be more than 2 unpaired segments; (iii) if only 2 segments are present, they must be unpaired. Tab. I exemplifies the constraints in more detail, for the simple case with coordination number equal to 4 , but generalizing to any coordination number is straightforward.
Constraint (i) is a simple connectivity constraint, stating that the chain does not terminate after a finite number of segments. Constraints (ii) and (iii) state that, if 2 unpaired segments come to a given site from different lattice bonds, they either pair each other (so that at least another bond is doubly occupied) or they are consecutive along the chain (all other bonds are empty). More precisely, constraint (ii) implies that unpaired chain stretches behave like self-avoiding walks, which cannot visit a lattice site more than once, unless they get paired. Constraint (iii) deserves some more discussion. A configuration with only two paired segments could represent a "terminal loop", in which the chain bends onto itself, to form a hairpin. In principle, such "zero-length loops" should be allowed by a basic 2-tolerant polymer model. Therefore, constraints (iii), which forbids them, can be considered either as a further detail, which defines a slightly different model, or as an approximation to the original one. Such approximation, which is conceptually independent of the subsequent approximate (Bethe) statistical treatment, has been firstly taken for technical reasons, in order to simplify the analytical calculations. We shall shortly discuss this technical issue in the following. By now, we only observe that there is actually a physical argument, which suggests that the modified model might be even a bit closer to the real system. In fact, for energetic reasons, terminal loops must have a minimum length of four bases, and experiments show that, in real RNA, typical hairpin loops are just of this kind (tetraloops) [13]. Therefore, a hairpin loop should have a finite, though small, entropy, which cannot be taken into account by a zero-length loop in the coarse-grained model.

Assuming a coordination number $k+1$, the Hamiltonian can be formally written as

$$
\mathcal{H}=\sum_{\left\{i_{0}, \ldots, i_{k}\right\}} H_{n_{i_{0}}, \ldots, n_{i_{k}}}+\sum_{i} h_{n_{i}},
$$

where the former sum runs over all sets of bonds $\left\{i_{0}, \ldots, i_{k}\right\}$ of all lattice sites, and the latter over all bonds $i$. Single-bond energy terms $h_{n}$ take into account pairing energies and chemical potential contributions, and can be defined as follows

$$
\begin{aligned}
& h_{0}=0, \\
& h_{1}=-\mu, \\
& h_{2}=-(\beta-\gamma)-2 \mu .
\end{aligned}
$$

Many-bond terms $H_{n_{0}, \ldots, n_{k}}$ take into account the constraints, assigning infinite energy penalties to forbidden configurations, and the stacking energy contributions. A definition of these terms would be quite cumbersome, from an analytical point of view, so that we can assume they are defined by a table like Tab. []

Let us briefly return to discuss constraint (iii), which, as previously mentioned, disallows zero-length hairpin loops, i.e., configurations with only two paired segments on the set of bonds of a given site. It turns out that, 
TABLE I: Configurations of a set of bonds of a given lattice site (left column); occupation numbers $n_{i}$ for each bond $i=0, \ldots, k$ (mid columns); total number of segments $N_{n_{0}, \ldots, n_{k}} \equiv \sum_{i=0}^{k} n_{i}$ and corresponding energy term $H_{n_{0}, \ldots, n_{k}}$ (right columns). Notice that: graphical representations are limited to four bonds; only configurations with even number of segments are reported, because odd numbers are forbidden (the corresponding energy terms are $\infty$ ); energy terms are invariant under bond permutations.

\begin{tabular}{|c|c|c|c|c|c|c|c|c|c|}
\hline conf. & $n_{0}$ & $n_{1}$ & $n_{2}$ & $n_{3}$ & $n_{4}$ & $\ldots$ & $n_{k}$ & $N_{n_{0}, \ldots, n_{k}}$ & $H_{n_{0}, \ldots, n_{k}}$ \\
\hline & 0 & 0 & 0 & 0 & 0 & $\ldots$ & 0 & 0 & 0 \\
\hline & 2 & 0 & 0 & 0 & 0 & $\ldots$ & 0 & 2 & $\infty$ \\
\hline & 1 & 1 & 0 & 0 & 0 & $\ldots$ & 0 & 2 & 0 \\
\hline & 2 & 2 & 0 & 0 & 0 & $\ldots$ & 0 & 4 & $-\gamma$ \\
\hline & 2 & 1 & 1 & 0 & 0 & $\ldots$ & 0 & 4 & 0 \\
\hline & 1 & 1 & 1 & 1 & 0 & $\ldots$ & 0 & 4 & $\infty$ \\
\hline & 2 & 2 & 2 & 0 & 0 & $\ldots$ & 0 & 6 & 0 \\
\hline & 2 & 2 & 1 & 1 & 0 & $\ldots$ & 0 & 6 & 0 \\
\hline & 2 & 2 & 2 & 2 & 2 & $\ldots$ & 2 & $2(k+1)$ & 0 \\
\hline
\end{tabular}

if we allowed such a configuration, local constraints of the form $H_{n_{0}, \ldots, n_{k}}$ would also allow, for instance, configurations with two paired segments disconnected from everything else, or even paired stretches of any length. In this way, we would not study an infinitely long polymer, but a mixture of polymers of different lengths, and this would be a completely different system. In order to avoid constraint (iii), we would have to exclude the aforementioned undesired configurations, and we would need a more complicated treatment. This will be the subject of a future work.

Let us now introduce the Bethe approximation. Basically, it consists in replacing the single walk on the regular lattice by a gas of walks, with the same selfavoidance constraints and interactions, on a Bethe lattice, having the same coordination number as the regular lattice one. In older literature, a Bethe lattice was simply understood to be the inner region of an infinite

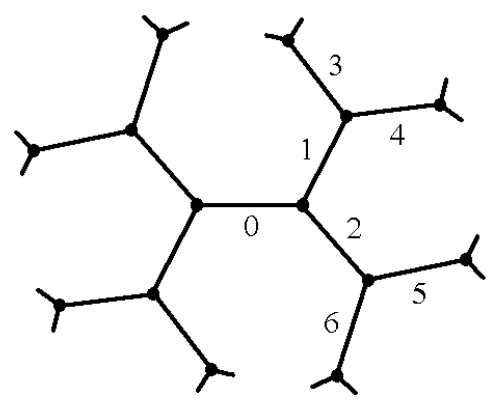

FIG. 1: Sketch of a Bethe lattice with $k=2$.

Cayley tree [28]. Nevertheless, it has been subsequently shown that the thermodynamic behavior of a Cayley tree is strongly affected by the presence of a boundary, and the exact solution for this system does not agree with the Bethe approximation [29]. More recently, it has been recognized that the Bethe lattice has to be a homogeneous, boundary-less structure (so that the thermodynamic properties of the system can be worked out by suitable self-consistence equations [5]), which also allows for the presence of macroscopically large loops [30]. The Bethe lattice is thus better defined as an ensemble of random graphs with fixed coordination number [31], which are locally treelike (in the sense that loop length is $\mathcal{O}(\ln \mathcal{N})$, where $\mathcal{N}$ is the number of nodes), and whose thermodynamic behavior is governed by the variational Bethe free energy [4]. The free energy minima can still be determined by solving a suitable recursion relation for the so-called partial partition functions [5, 32]. We shall address this issue in Appendix $\mathrm{A}$. Hereafter, we just give an intuitive derivation, based on the treelike nature of the system. Let us consider for instance the Bethe lattice depicted in Fig. 1, and the right part of the system, starting with the bond denoted by 0 . Since loops connecting the two parts are (with high probability) infinitely long in the thermodynamic limit, we can imagine that the two parts are actually disconnected branches and that we can thus define a partial Hamiltonian, obtained by Eq. (11) restricting the sum to bond variables in one branch. The corresponding partial partition function $W_{n_{0}}$ can be computed by summing the Boltzmann weights of the partial Hamiltonian over the configurations of the branch except the 0 bond. Actually, it is convenient to work with a normalized partial partition function $w_{n_{0}} \propto W_{n_{0}}$, such that

$$
\sum_{n_{0}=0}^{2} w_{n_{0}}=1 .
$$

The normalized partial partition function $w_{n_{0}}$ represents, as a function of $n_{0}$, the probability distribution of the configuration variable "in the absence of the other branch". Let us now observe that, in the thermodynamic limit, and in the hypothesis of a homogeneous system, the subbranches attached to the 0 bond should be equivalent 
to main one, so that one can write the recursion equation

$$
w_{n_{0}}=q^{-1} e^{-h_{n_{0}}} \sum_{n_{1}=0}^{2} \ldots \sum_{n_{k}=0}^{2} e^{-H_{n_{0}, n_{1}, \ldots, n_{k}}} \prod_{i=1}^{k} w_{n_{i}} .
$$

The sum runs over configuration variables of bonds attached to the 0 bond ( $n_{1}$ and $n_{2}$, in our example), the energy terms $h_{n_{0}}$ and $H_{n_{0}, n_{1}, \ldots, n_{k}}$ are assumed to be normalized to temperature, and $q$ is a normalization constant, imposed by Eq. (5). A more explicit form of the recursion equation is given in Appendix $B$, where the specific energy terms $H_{n_{0}, n_{1}, \ldots, n_{k}}$ of our model are taken into account. The recursion equation can be solved numerically by a simple iterative algorithm. All equilibrium properties of the system can be derived from the knowledge of the partial partition function.

First of all, we can compute the probability distribution $p_{n}$ of a bond configuration variable $n$, by considering the operation of attaching 2 branches to the given bond. We obtain

$$
p_{n}=z^{-1} e^{h_{n}} w_{n}^{2}
$$

where

$$
z=\sum_{n=0}^{2} e^{h_{n}} w_{n}^{2}
$$

provides normalization. The average number of segments per bond, which we shall briefly refer to as density in the following, can be evaluated as

$$
\rho=\sum_{n=0}^{2} n p_{n}=p_{1}+2 p_{2}
$$

The density $\rho$ is the main order parameter for our system. As a secondary order parameter, we evaluate the fraction of paired segments

$$
\phi=2 p_{2} / \rho .
$$

The grand-canonical free energy (grand-potential) per bond $\omega$ can be determined as

$$
\omega=-\frac{2 \ln q-(k-1) \ln z}{k+1},
$$

where $q$ is the normalization constant of the recursion equation (6) and $z$ is given by Eq. (8). The derivation of this expression requires some manipulations and is reported in Appendix $\mathrm{A}$. From the knowledge of the grandpotential one can derive all other thermodynamic properties, and determine thermodynamic stability for each phase.

\section{THE PHASE DIAGRAM AND THE SINGLE-MOLECULE LIMIT}

In the framework of a grand-canonical formulation, the phase diagram can be described as a function of temperature and chemical potential. For a polymer, the latter controls the average chain length. For example, in
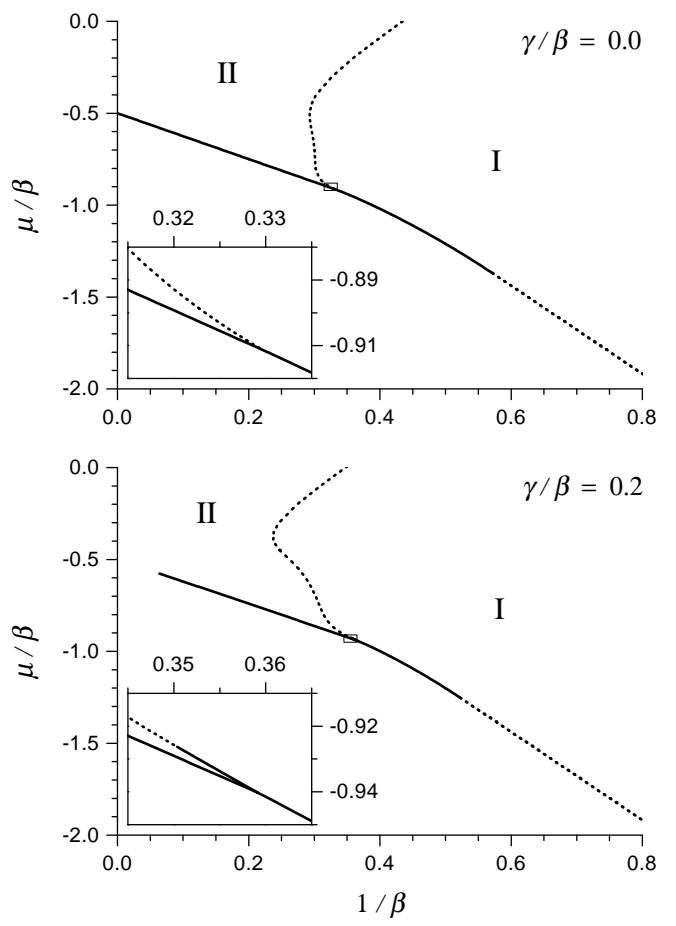

FIG. 2: Chemical potential-temperature $(\mu / \beta$ vs $1 / \beta)$ phase diagram for the zero stacking case $(\gamma=0$, upper graph) and a nonzero stacking case $(\gamma / \beta=0.2$, lower graph). Solid lines denote first order transitions; dashed lines denote second order transitions. The ordinary dense phase is denoted by I, whereas the fully paired dense phase is denoted by II. The zero density phase is left blank. The insets display the regions enclosed in the small rectangles.

the simple $\Theta$ model, there exists a phase transition line $\mu=\varphi(T)$ at which (for increasing $\mu$ values) the average length either diverges continuously (for temperatures higher than some temperature $\Theta$ ) or jumps discontinuously to infinity (for temperatures lower than $\Theta$ ) [1]. The transition line is identified as the thermodynamic limit of a single chain, so that we denote it as the "singlemolecule" limit. Alternatively, the system can be described in terms of a segment density $\rho$, and one obtains $\rho=0$ for $\mu<\varphi(T)$ and $\rho>0$ for $\mu>\varphi(T)$. The transition is second order for $T>\Theta$ and first order for $T<\Theta$. In the limit $\mu \rightarrow \varphi(T)^{+}$, the properties of the dense phase approach those of a single chain, and, in particular, the segment density $\rho$ is a measure of the chain compactness. Therefore, the tricritical point $(\Theta, \varphi(\Theta))$, known as $\Theta$ point, represents a coil-globule collapse.

We present grand-canonical phase diagrams of our Bethe lattice model for the case of zero stacking effect $(\gamma=0)$ and for a case of nonzero stacking effect $(\gamma / \beta=0.2)$, which show qualitatively different behaviors. Let us recall that $\gamma / \beta$ quantifies the ratio between the neat stacking energy $\gamma$ and the total effect of pairing and stacking (the simple pairing energy is $\beta-\gamma$ ). We shall shortly denote $\gamma / \beta$ as stacking ratio in the following. We set $k=11$ (coordination number $=12$ ), expect- 
ing to approximate the fcc lattice. Let us consider the zero stacking case first. The phase diagram is displayed in Fig. 2 (upper graph), where the temperature variable is $1 / \beta$ and the chemical potential variable is $\mu / \beta$. We find three different phases: a zero density phase $(\mathrm{O})$, an ordinary dense phase (I), and a fully paired dense phase (II). The zero density phase is characterized by $\rho=0$. Since only a vanishing fraction of bonds is occupied in this phase, also the grand-potential per bond $\omega$ vanishes, and, for the same reason, the fraction of paired segments $\phi$ is undefined. The I phase is characterized by $0<\rho<2$ and $0<\phi<1$, i.e., it is a dense phase which possesses a finite fraction of paired segments. We can roughly compare it to the dense phase of an ordinary $\Theta$ model. Finally, the II phase is characterized by $0<\rho<2$ and $\phi=1$, i.e., it is a dense phase in which every segment is paired.

The transition line between the $\mathrm{O}$ and I phases turns out to be partially first and partially second order. The two regimes are separated by a tricritical point. As explained in Appendix [C] it is possible to determine analytically the equation of the second order line,

$$
\mu=-\ln k,
$$

and the location of the tricritical point

$$
\beta=\ln \frac{k^{2}}{k+(k-1) e^{-\gamma}} .
$$

It has been pointed out that, for this kind of (2-tolerant) polymer models, the tricritical point exhibits peculiar values of the critical exponents, that are different from those of the ordinary self-avoiding walk with attractive interaction, and suggest a linear-to-branched polymer transition [33]. Evidences of such a behavior will be observed also in our model. Nevertheless, since this point still corresponds to a continuous collapse and, since exponent differences cannot be detected at a Bethe approximation level, we shall all the same speak of a $\Theta$ point in the following.

In the dense region $(\rho>0)$ a second order transition line separates the I and II phases. This line joins to the transition line with the $\mathrm{O}$ phase at a critical end-point. The latter corresponds to another continuous conformational transition for the single molecule. The behavior is different, in the presence of the stacking effect, as shown in Fig. 2 (lower graph). The same three phases O, I, and II discussed above are present, and also the high temperature region of the phase diagram is qualitatively similar, although we can observe a lower $\Theta$ temperature, in agreement with Eq. (13). On the contrary, the I-II transition line turns out to be partially second and partially first order, giving rise to a tricritical point in the dense region. In this way, the critical end-point disappears, and is replaced by a triple point, which corresponds to a discontinuous transition in the single-molecule limit.

Let us now investigate this limit in more detail. First of all, we consider the fraction of paired segments $\phi$, computed for $\mu$ tending to the transition line from above,

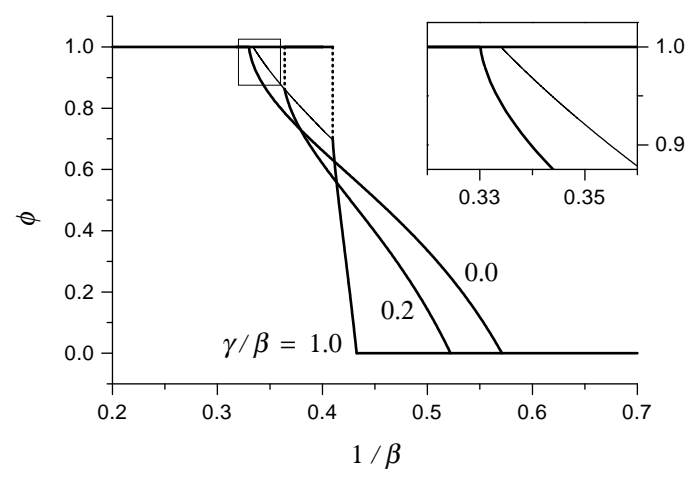

FIG. 3: Fraction of paired segments as a function of temperature $(\phi$ vs $1 / \beta)$ in the single-molecule limit, for different values of the stacking ratio $\gamma / \beta$. Dashed lines denote discontinuities; a thin solid line connects transition values. The inset displays the region enclosed in the small rectangle.

as a function of temperature. The results are reported in Fig. 3, for three different values of the stacking ratio $\gamma / \beta=0,0.2,1$. For all cases, we can see that $\phi$ is rigorously zero above the $\Theta$ temperature. In this regime, which we can denote as coil state, the polymer behaves like an ordinary self-avoiding walk without self-interaction. Upon decreasing temperature below the $\Theta$ point, the fraction of paired segments begins to increase, revealing formation of contacts. We can identify this regime as the molten state. As previously mentioned, the $\Theta$ temperature decreases, upon increasing the stacking energy. Upon further decreasing temperature, $\phi$ reaches the saturation value $\phi=1$. In this regime, which we simply denote as paired state since all segments are paired, we can imagine our system as a branched double chain. In this sense, we can identify this phase as a "native" RNA-like state, although it does not at all correspond to a single configuration, as it will become clearer later. As previously mentioned, the molten-paired transition is continuous in the zero stacking case, but becomes first order in the nonzero stacking cases. More precisely, we observe that the stacking energy needed to change the order of the transition is very small but finite, as suggested in Fig. 3 by the thin line connecting the transition values of $\phi$.

We also investigate the temperature dependence of the entropy per segment, which can be computed as follows. The grand-potential per bond can be written as

$$
\omega=f-\mu \rho,
$$

where $f$ is the Helmholtz free energy per bond. As previously mentioned, $\omega$ vanishes at the $\mathrm{O}$ phase boundary, so that in this case $\mu$ coincides with the Helmholtz free energy per segment

$$
\mu=f / \rho .
$$

Remembering that our energies are normalized to temperature, the equation of the $\mathrm{O}$ phase boundary can be 


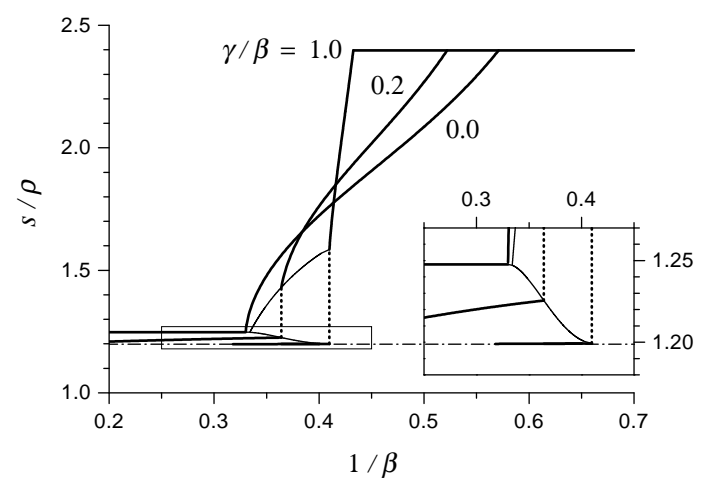

FIG. 4: Entropy per segment as a function of temperature $(s / \rho$ vs $1 / \beta)$ in the single-molecule limit, for different values of the stacking ratio $\gamma / \beta$. Dashed lines denote discontinuities; thin solid lines connect transition values. The inset displays the region enclosed in the small rectangle. A dash-dotted line indicates the entropy value $\ln k / 2$.

written as

$$
\mu / \beta=\varphi(1 / \beta),
$$

where the function $\varphi$ is known numerically with high precision. The entropy per segment (in natural units) can thus be easily determined as

$$
s / \rho=-\varphi^{\prime}(1 / \beta),
$$

where $\varphi^{\prime}$ denotes the first derivative of $\varphi$. We report the results in Fig. 4 for the usual values of the stacking ratio $\gamma / \beta=0,0.2,1$. For all cases, in the coil state, the entropy per segment is rigorously independent of temperature and equal to $\ln k$, as it can be easily derived by Eqs. (16), (12) and (17). This value characterizes an ordinary self-avoiding walk on the Bethe lattice [9]. In the molten state, the entropy starts decreasing (as temperature decreases), more and more rapidly, upon increasing the stacking effect. Finally, in the paired state, the entropy is almost constant and its value turns out to be slightly larger than $(\ln k) / 2$, which would characterize a self-avoiding double chain. The excess entropy with respect to this value, which is due to branching, tends to zero as temperature goes to zero, and decreases upon increasing the stacking effect.

According to the results reported so far, the low temperature phase might appear as almost completely quenched. The following analysis of the average length of double chain stretches (which we shall shortly refer to as stacking length in the following) demonstrates that this is not the case. Let $\pi$ denote the probability that, given a lattice bond occupied by two paired segments, just one neighbor bond (in a given direction) is occupied by two paired segments as well. We can call $\pi$ the stacking probability. Due to the Markovian nature of the Bethe lattice, the probability of having a double chain stretch of length $l$ in the given direction is $\pi^{l-1}(1-\pi)$.

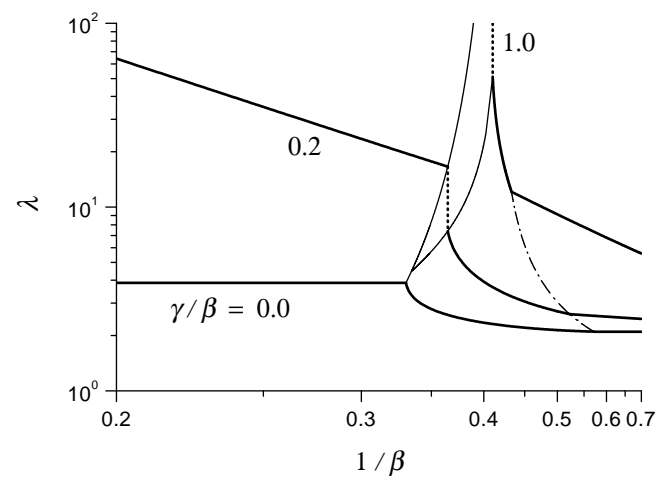

FIG. 5: Average stacking length as a function of temperature ( $\lambda$ vs $1 / \beta)$ in the single-molecule limit, for different values of the stacking ratio $\gamma / \beta$. Dashed lines denote discontinuities; thin solid lines connect transition values; a dash-dotted line, determined by Eqs. (18) and (21), connects $\Theta$-point values.

The average stacking length is therefore

$$
\lambda=\sum_{l=1}^{\infty} l \pi^{l-1}(1-\pi)=\frac{1}{1-\pi} .
$$

Considering the recursion equation (B3), we can derive the stacking probability as the ratio between the weight of the stacked configuration $\left(\begin{array}{l}k \\ 1\end{array}\right) e^{\gamma} w_{2} w_{0}^{k-1}$ and the total weight of the configurations compatible with the two paired segments (coinciding with the left-hand side, at a fixed point of the recursion equations). Remembering also the expression (4) for $h_{2}$, we easily obtain

$$
\pi=q^{-1} k e^{2 \mu+\beta} w_{0}^{k-1},
$$

where of course $q$ and $w_{0}$ are available from the numerical solution. It is also useful to derive an explicit expression for $\pi$ at the second order O-I phase boundary, in order to avoid taking limits numerically. Performing basically the same calculation with Eq. (C2), taking the limit $x, y \rightarrow 0$, and making use of Eq. (C6), we obtain

$$
\pi=\frac{k}{k+(k-1) e^{-\gamma}} .
$$

Moreover, comparing this equation with Eq. (13), we obtain, at the $\Theta$ point, the following simple relation

$$
\pi=e^{\beta} / k .
$$

The results are reported in Fig. 5, again for $\gamma / \beta=$ $0,0.2,1$. The most interesting features appear in the coil and paired states. In particular, we can observe that the stacking length is constant with respect to temperature if $\gamma=0$, i.e., in the absence of the stacking effect. On the contrary, even a very small stacking energy makes the stacking length increase upon decreasing temperature. Since the length scale is logarithmic and the temperature scale is "inverse", straight lines indicate that $\lambda$ is exponential in $\beta$ in these phases. As a consequence, 


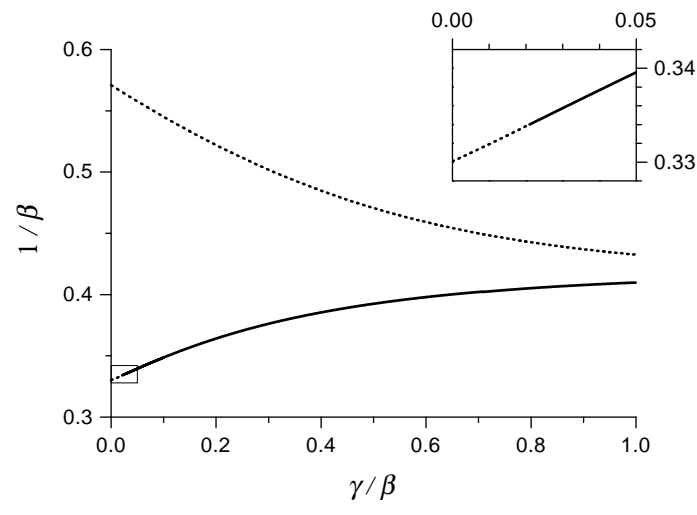

FIG. 6: Transition temperatures as a function of the stacking ratio $(1 / \beta$ vs $\gamma / \beta)$ in the single-molecule limit. Solid lines denote first order transitions; dashed lines denote second order transitions. The inset displays the region enclosed in the small rectangle.

in the presence of the stacking effect, the stacking length diverges as temperature goes to zero, so that the ground state of the model can be thought of as a unique double chain (hairpin). Let us also notice that, in the coil state, the stacking length does not vanish at any finite temperature value, unlike the fraction of paired segments. These results do not disagree, meaning that, if a (rare) contact is formed, it has nevertheless a probability of not being isolated.

We have already described the overall behavior of the phase transitions in the single-molecule limit, as a function of the stacking ratio. As this parameter increases, the $\Theta$ temperature decreases, whereas the molten-paired transition temperature increases, and the transition changes from second to first order. For the sake of completeness, we report in Fig. 6 the transition temperatures as a function of the stacking ratio. The $\Theta$ transition line is given analytically by Eq. (13), whereas the molten-paired transition has been determined numerically. In the latter transition, we recover the continuous regime at very low stacking values and the discontinuous regime at higher stacking values. Quantitatively, the boundary between the two regimes is found to occur at $\gamma / \beta \approx 0.0210$.

\section{DISCUSSION AND CONCLUSIONS}

In this paper, we have investigated a 2-tolerant polymer model on the Bethe lattice, with both contact and stacking interactions. The model is expected to mimic some qualitative features of conformational transitions of RNA molecules. The most striking results are the presence of a low temperature transition to a fully-paired phase and the effect of stacking, which turns out to drive such transition from second to first order. We have already questioned, throughout the paper, whether these results have some relevance to the denaturation transition of real RNA. The most important warning concerns the fact that we completely neglect chemical heterogeneities, which are indeed present in RNA. As a consequence, we observe that the fully-paired state does not correspond to a well defined secondary structure, but indeed to a variety of structures. On the contrary, several investigations proposed in the literature take into account randomly distributed heterogeneous sequences 21, 22, 23, 24, 27]. Even in this case, the low temperature glasslike phase does not correspond to a fixed structure, but the general claim is that it could describe average RNA properties. On this kind of models, the only work we are aware of, which performs a systematic investigation as a function of the strength of the stacking interaction, is one by Burghardt and Hartmann [27]. In the cited paper, the authors do not find any evidence of a change in the order of the (temperatureinduced) denaturation transition. Nevertheless, in a previous work, Zhou and Zhang [34] had observed that an increasing stacking energy could change the order of a force-induced denaturation. This result has been actually criticized by Müller [26], who argued that the apparent first order transition was rather to be interpreted as a sharp cross-over. It is important to notice, however, that all the cited investigations neglect the effect of excluded volume. On the contrary, the Bethe lattice approximation is partially able to account for excluded volume, by imposing local self-avoidance constraints. This may be indeed a reason for our qualitatively different results. In order to investigate this issue in more detail, it would be interesting to extend the Bethe lattice analysis to the case of a random heterogeneous sequence, making use the recently proposed cavity method [31], along the lines traced by Montanari, Müller, and Mezard, for the selfavoiding heteropolymer [35, 36].

Let us also compare our results with the Monte Carlo simulations by Baiesi, Orlandini, and Stella [11]. In the cited work, the authors investigate a 2-tolerant trail with contact energy only, on the fcc lattice, fully taking into account excluded volume. As the polymer is assigned an orientation (whence the term "trail"), only antiparallel contacts are allowed. Let us notice, by the way, that in our treatment we have not introduced orientation explicitly. Nevertheless, it is possible to show that the latter, together with the constraint on antiparallel contacts, are equivalent to a simple renormalization of the partial partition functions, with no effect on observable quantities. Of course, we cannot expect that any result concerning critical exponents could be reproduced in the framework of our mean-field-like approach. Nevertheless, it is noticeable that, for $k=11$ (fcc lattice) and $\gamma=0$ (contact energy only), we predict a $\Theta$ temperature $(\beta \approx 1.7513)$ not so far from the Monte Carlo result $(\beta \approx 1.9)$. Apart from this result, the fact itself that we observe a $\Theta$-like transition may rise some interest. Indeed, an important result of Ref. 11 is that, if pseudoknots (i.e., tertiary RNA structure) are forbidden, then the $\Theta$-like transition is replaced by a smooth cross-over. We wonder how the 
Bethe lattice approach can reproduce (at least qualitatively) the situation with pseudoknots, since the model is embedded on a treelike structure. Our tentative explanation is based on the previously mentioned definition of the Bethe lattice in terms of random graphs [31], rather than the more usual and quick definition ("the infinite interior of the infinite Cayley tree" [5]). In the former picture, pseudoknots could be realized via large loops present in the graphs, although in the current treatment we do not have any control on them. Let us finally notice that, conversely, just the presence of only large loops in the Bethe lattice might create some artifact in the description of RNA statistics, especially with a constraints which disallows zero-length hairpin turns, as discussed in Sec. III. We find these issues worth a deeper investigation, which we would like to devote a future work to.

\section{APPENDIX A: BETHE FREE ENERGY}

In this appendix we first give a derivation of the recursion equation (6) as a stationarity condition for the variational Bethe free energy, and then prove the validity of the expression (11) for the equilibrium free energy.

Let us consider a Bethe lattice with $c$ coordination number, and assume that a configuration variable $n_{i}$ is associated to each lattice bond $i$. Let us also assume that the Hamiltonian of the system is the one given in Eq. (1), which includes couplings among the $c$ bonds of each site. Let the coupling terms be invariant under permutation of the configuration variables. Expecting a homogeneous thermodynamic state (i.e., that all local probability distributions are equal), we write the Bethe free energy per site as

$F=\sum_{\left\{n_{i}\right\}} P_{\left\{n_{i}\right\}}\left(H_{\left\{n_{i}\right\}}+\ln P_{\left\{n_{i}\right\}}\right)+\frac{c}{2} \sum_{n} p_{n}\left(h_{n}-\ln p_{n}\right)$

where $\left\{n_{i}\right\}$ stands for $n_{1}, \ldots, n_{c}$, while $p_{n}$ and $P_{\left\{n_{i}\right\}}$ denote respectively the single-variable and the $c$-variable probability distributions. Accordingly, $\sum_{n}$ and $\sum_{\left\{n_{i}\right\}}$ denote the sums over possible values of the configuration variables. Let us notice that, as far as the entropic part is concerned, the latter term of the Bethe free energy can be thought of as a correction over the former term, such that the mean field free energy is recovered, when the joint probability distribution factorizes. Equilibrium probability distributions can be determined as minima of the Bethe free energy, satisfying suitable normalization and compatibility constraints. By "compatibility", we mean that marginalizations of the joint probability distribution must give the single-variable distribution, according to the relations

$$
p_{n_{i}}=\sum_{\left\{n_{j}\right\}_{j \neq i}} P_{\left\{n_{j}\right\}} \quad i=1, \ldots, c,
$$

where the sum runs over possible values of the configuration variables $n_{1}, \ldots, n_{c}$, except $n_{i}$. We thus have a con- strained optimization problem, for which, in the framework of the Lagrange multiplier method, we can solve analytically stationarization with respect to probability distributions. Doing so, the latter can be written as a function of suitable variables $z, Z$, and $w_{n}$, which correspond to Lagrange multipliers, and are to be determined in order to satisfy the constraints. We obtain

$$
\begin{aligned}
p_{n} & =z^{-1} e^{h_{n}} w_{n}^{2}, \\
P_{\left\{n_{i}\right\}} & =Z^{-1} e^{-H_{\left\{n_{i}\right\}}} \prod_{i=1}^{c} w_{n_{i}} .
\end{aligned}
$$

Let us notice that $w_{n}$ plays the role of the (normalized) partial partition function introduced in the text, whereas the two constants $z$ and $Z$, associated to the normalization constraints, are easily determined as a function of $w_{n}$. Moreover, imposing the compatibility constraints (A2), one obtains the following recursion equation

$$
w_{n_{i}}=q^{-1} e^{-h_{n_{i}}} \sum_{\left\{n_{j}\right\}_{j \neq i}} e^{-H_{\left\{n_{j}\right\}}} \prod_{\substack{j=1 \\ j \neq i}}^{c} w_{n_{j}},
$$

where

$$
q=Z / z
$$

It is possible to show that, because of a slight redundance of the constraints, one can choose the constant $q$ at each iteration in an arbitrary way, for instance by imposing the normalization condition $\sum_{n} w_{n}=1$, without affecting "observable" quantities. Let us also notice that the $c$ compatibility conditions (A2) would require in principle $c$ sets of "Lagrange multipliers" $w_{n}^{(i)}$, for $i=1, \ldots, c$. Nevertheless, one can show that, due to invariance of $H_{\left\{n_{i}\right\}}$ under permutation, all the sets must be equal to a single one, which we have just denoted as $w_{n}$.

Let us now derive the simple free energy formula (11) presented in the text. Let us plug the expressions (A3) and (A4) for the equilibrium probability distributions into the logarithmic terms of the variational free energy (A1). By simple algebra, we obtain

$$
\begin{aligned}
F= & -\left(\sum_{\left\{n_{i}\right\}} P_{\left\{n_{i}\right\}}\right) \ln Z+\frac{c}{2}\left(\sum_{n} p_{n}\right) \ln z \\
& -\sum_{i=1}^{c} \sum_{n_{i}}\left(p_{n_{i}}-\sum_{\left\{n_{j}\right\}_{j \neq i}} P_{\left\{n_{j}\right\}}\right) \ln w_{n_{i}} .
\end{aligned}
$$

Since at equilibrium the normalization and compatibility constraints are satisfied, the previous expression immediately simplifies to

$$
F=-\ln Z+\frac{c}{2} \ln z
$$

Taking into account that there are $c / 2$ bonds per site, that $c=k+1$, and making use of Eq. (A6), we finally obtain Eq. (11) for the equilibrium free energy per bond. 


\section{APPENDIX B: RECURSION EQUATIONS}

In this appendix we write an explicit form for the recursion equations (6), introducing the values of the couplings $H_{n_{0}, \ldots, n_{k}}$, determined according to criteria explained in Tab. [I Moving the constant $q$ and the singlevariable energies $h_{n}$ to the left-hand sides, and remembering that $h_{0}=0$, we obtain

$$
\begin{aligned}
q w_{0}= & \sum_{\substack{m=0 \\
m \neq 1,2}}^{k}\left(\begin{array}{c}
k \\
m
\end{array}\right) w_{2}^{m} w_{0}^{k-m}+\left(\begin{array}{l}
k \\
2
\end{array}\right) e^{\gamma} w_{2}^{2} w_{0}^{k-2} \\
& +\left(\begin{array}{c}
k \\
2
\end{array}\right) w_{1}^{2} \sum_{m=0}^{k-2}\left(\begin{array}{c}
k-2 \\
m
\end{array}\right) w_{2}^{m} w_{0}^{k-2-m}, \quad(\mathrm{~B} 1) \\
q e^{h_{1}} w_{1}= & \left(\begin{array}{c}
k \\
1
\end{array}\right) w_{1} \sum_{m=0}^{k-1}\left(\begin{array}{c}
k-1 \\
m
\end{array}\right) w_{2}^{m} w_{0}^{k-1-m}, \\
q e^{h_{2}} w_{2}= & \sum_{m=2}^{k}\left(\begin{array}{c}
k \\
m
\end{array}\right) w_{2}^{m} w_{0}^{k-m}+\left(\begin{array}{c}
k \\
1
\end{array}\right) e^{\gamma} w_{2} w_{0}^{k-1} \\
& +\left(\begin{array}{c}
k \\
2
\end{array}\right) w_{1}^{2} \sum_{m=0}^{k-2}\left(\begin{array}{c}
k-2 \\
m
\end{array}\right) w_{2}^{m} w_{0}^{k-2-m} .
\end{aligned}
$$

Let us give a physical explanation of the various terms appearing on the right-hand sides. They have to take into account all the allowed configurations of $k$ bonds sharing one site with a given bond, whose configuration is fixed ( $n=0,1,2$ for the three equations, respectively). In the first equation, the fixed configuration is $n=0$ (empty bond). In the right-hand side, the first two terms refer to configurations with $m=0, \ldots, k$ bonds occupied by paired segments, and $k-m$ empty bonds. As explained in the text, the case $m=1$ is forbidden, and the case $m=2$ is treated separately, since it has to take into account a stacking energy contribution. The third term deals with the case of 2 bonds occupied by unpaired segments, $m=0, \ldots, k-2$ by paired segments, and $k-2-m$ empty bonds. In the second equation, the fixed bond configuration is $n=1$ (bond occupied by an unpaired segment). Therefore, in the right-hand side, there is always one bond occupied by an unpaired segment, together with $m=0, \ldots, k-1$ occupied by paired segments, and $k-1-m$ empty bonds. In the third equation, the fixed configuration is $n=2$ (bond occupied by paired segments). In the right-hand side, the first two terms refer to configurations with $m=0, \ldots, k$ bonds occupied by paired segments, and $k-m$ empty bonds. As in the first equation, the case $m=0$ is forbidden, and the case $m=1$ is treated separately, because of the stacking energy contribution. The third term deals with 2 bonds occupied by unpaired segments, $m=0, \ldots, k-2$ by paired segments, and $k-2-m$ empty bonds.

The above form of the recursion equations can be further simplified, making use of the binomial expansion.
By simple algebra, we finally obtain

$$
\begin{aligned}
q w_{0}= & \left(w_{0}+w_{2}\right)^{k}+\left(\begin{array}{l}
k \\
2
\end{array}\right) w_{1}^{2}\left(w_{0}+w_{2}\right)^{k-2} \\
& -\left(\begin{array}{c}
k \\
1
\end{array}\right) w_{2} w_{0}^{k-1}+\left(\begin{array}{l}
k \\
2
\end{array}\right)\left(e^{\gamma}-1\right) w_{2}^{2} w_{0}^{k-2}, \\
q e^{h_{1}} w_{1}= & \left(\begin{array}{c}
k \\
1
\end{array}\right) w_{1}\left(w_{0}+w_{2}\right)^{k-1}, \\
q e^{h_{2}} w_{2}= & \left(w_{0}+w_{2}\right)^{k}+\left(\begin{array}{l}
k \\
2
\end{array}\right) w_{1}^{2}\left(w_{0}+w_{2}\right)^{k-2} \\
& -w_{0}^{k}+\left(\begin{array}{c}
k \\
1
\end{array}\right)\left(e^{\gamma}-1\right) w_{2} w_{0}^{k-1} .
\end{aligned}
$$

\section{APPENDIX C: THETA POINT}

Hereafter, we report the derivation of Eq. (13), i.e., the analytical expression for the $\Theta$ transition temperature. Eq. (12), i.e., the second order O-I phase boundary, comes out as a by-product of this derivation. Let us first define the ratios $x \equiv w_{1} / w_{0}$ and $y \equiv w_{2} / w_{0}$, for which we can easily derive two recursive equations from (B4), (B5), and (B6)

$x=e^{-h_{1}} \frac{\left(\begin{array}{l}k \\ 1\end{array}\right) x(1+y)^{k-1}}{d}$,
$y=e^{-h_{2}} \frac{(1+y)^{k}+\left(\begin{array}{l}k \\ 2\end{array}\right) x^{2}(1+y)^{k-2}-1+\left(\begin{array}{l}k \\ 1\end{array}\right)\left(e^{\gamma}-1\right) y}{d}$,

where

$d \equiv(1+y)^{k}+\left(\begin{array}{l}k \\ 2\end{array}\right) x^{2}(1+y)^{k-2}-\left(\begin{array}{c}k \\ 1\end{array}\right) y+\left(\begin{array}{l}k \\ 2\end{array}\right)\left(e^{\gamma}-1\right) y^{2}$.

From (C1) and (C3), assuming that $x \neq 0$, i.e., that we are in the I phase, we obtain

$\left(\begin{array}{l}k \\ 2\end{array}\right) x^{2}=\left(\begin{array}{l}k \\ 1\end{array}\right) e^{\mu}(1+y)-(1+y)^{2}+\frac{\left(\begin{array}{l}k \\ 1\end{array}\right) y+\left(\begin{array}{l}k \\ 2\end{array}\right)\left(e^{\gamma}-1\right) y^{2}}{(1+y)^{k-2}}$.

Moreover, in the $y \rightarrow 0$ limit, i.e., very close to the boundary with the $\mathrm{O}$ phase, we can write

$$
\left(\begin{array}{l}
k \\
2
\end{array}\right) x^{2}=\left(k e^{\mu}-1\right)+\left(k e^{\mu}+k-2\right) y+\mathcal{O}\left(y^{2}\right) .
$$

Since we have observed from the numerics that such boundary is second order, we have that $y \rightarrow 0$ should imply also $x \rightarrow 0$. We then argue that the zeroth order term on the right-hand side of the previous equation must vanish. In this way we obtain Eq. (12). Plugging this equation into the previous one, we obtain

$$
\left(\begin{array}{l}
k \\
2
\end{array}\right) x^{2}=(k-1) y+\mathcal{O}\left(y^{2}\right),
$$


whereas, remembering also Eq. (4), Eq. (C2) becomes

$$
y=e^{\beta} \frac{k\left(e^{\gamma}+1\right)-1}{k^{2} e^{\gamma}} y+\mathcal{O}\left(y^{2}\right) .
$$

Now, in order to allow the possibility that, along the phase boundary, there can exist some point in which $y$ is vanishingly small but not zero (i.e., a tricritical point), we have to equate the first order coefficients on the two sides of the previous equation. By simple algebra, we obtain the $\Theta$ point condition (13).
[1] C. Vanderzande, Lattice Models of Polymers (Cambridge University Press, Cambridge, 1998).

[2] P. G. de Gennes, Scaling Concepts in Polymer Physics (Cornell University Press, Ithaca, 1988).

[3] H. A. Bethe, Proc. R. Soc. A 150, 552 (1935).

[4] R. Kikuchi, Phys. Rev. 81, 988 (1951).

[5] P. D. Gujrati, Phys. Rev. Lett. 74, 809 (1995).

[6] P. D. Gujrati, Phys. Rev. Lett. 74, 1367 (1995).

[7] P. D. Gujrati, J. Chem. Phys. 108, 5089 (1998).

[8] P. D. Gujrati, J. Chem. Phys. 108, 5104 (1998).

[9] S. Lise, A. Maritan, and A. Pelizzola, Phys. Rev. E 58, R5241 (1998).

[10] C. Buzano and M. Pretti, J. Chem. Phys. 117, 10360 (2002).

[11] M. Baiesi, E. Orlandini, and A. L. Stella, Phys. Rev. Lett. 91, 198102 (2003).

[12] P. Leoni and C. Vanderzande, Phys. Rev. E 68, 051904 (2003).

[13] I. Tinoco and C. Bustamante, J. Mol. Biol. 293, 271 (1999).

[14] R. Turner, Nature 418, 213 (2002).

[15] J. A. Doudna and T. R. Cech, Nature 418, 222 (2002).

[16] P. B. Moore and T. A. Steitz, Nature 418, 229 (2002).

[17] J. Sponer, J. Leszczynski, and P. Hobza, Biopolymers 61, 3 (2001).

[18] I. Tazawa, T. Koike, and Y. Inoue, European Journal of Biochemistry 109, 33 (1980).

[19] E. C. Chen, E. S. Chen, and W. E. Wentworth, Biochem. Biophys. Res. Commun. 171, 97 (1990).
[20] R. I. Dima, C. Hyeon, and D. Thirumalai, J. Mol. Biol. 347, 53 (2005).

[21] R. Bundschuh and T. Hwa, Phys. Rev. Lett. 83, 1479 (1999).

[22] R. Bundschuh and T. Hwa, Phys. Rev. E 65, 031903 (2002).

[23] A. Pagnani, G. Parisi, and F. Ricci-Tersenghi, Phys. Rev. Lett. 84, 2026 (2000).

[24] E. Marinari, A. Pagnani, and F. Ricci-Tersenghi, Phys. Rev. E 65, 041919 (2002).

[25] R. Mukhopadhyay, E. Emberly, C. Tang, and N. S. Wingreen, Phys. Rev. E 68, 041904 (2003).

[26] M. Müller, Phys. Rev. E 67, 021914 (2003).

[27] B. Burghardt and A. K. Hartmann, Phys. Rev. E 71, 021913 (2005).

[28] C. Domb, Adv. Phys. 9, 145 (1960).

[29] T. P. Eggarter, Phys. Rev. B 9, 2989 (1974).

[30] P. D. Gujrati and D. Bowman, J. Chem. Phys. 111, 8151 (1999).

[31] M. Mezard and G. Parisi, Eur. Phys. J. B 20, 217 (2001).

[32] M. Pretti, J. Stat. Phys. 111, 993 (2003).

[33] E. Orlandini, F. Seno, A. L. Stella, and M. C. Tesi, Phys. Rev. Lett. 68, 488 (1992).

[34] H. Zhou and Y. Zhang, J. Chem. Phys. 114, 8694 (2001).

[35] A. Montanari, M. Müller, and M. Mézard, Phys. Rev. Lett. 92, 185509 (2004).

[36] M. Müller, M. Mézard, and A. Montanari, J. Chem. Phys. 120, 11233 (2004). 